\title{
Effect of graphene addition on the transport critical current density of bulk $\left(\mathrm{Tl}_{0.85} \mathrm{Cr}_{0.15}\right)$ $\mathrm{Sr}_{2} \mathrm{CaCu}_{2} \mathrm{O}_{7-\delta}$ superconductor
}

\begin{abstract}
In this work, the effect of graphene addition on the transport critical current density of $\left(\mathrm{Tl}_{0.85} \mathrm{Cr}_{0.15}\right) \mathrm{Sr}_{2} \mathrm{CaCu}_{2} \mathrm{O}_{7-\delta}$ (Tl-1212) superconductor was investigated. Thallium-based high temperature superconductor (HTS) with nominal starting composition $\left(\mathrm{Tl}_{0.85} \mathrm{Cr}_{0.15}\right)$ $\mathrm{Sr}_{2} \mathrm{CaCu}_{2} \mathrm{O}_{7-\delta}$ was prepared using high purity oxide powders via solid state reaction method. $0.001-0.005 \mathrm{wt}$ \% of graphene were added into Tl-1212 superconductors. The characteristic of the samples were determined by powder X-ray diffraction method, scanning electron microscopy (SEM), energy dispersive X-Ray analysis (EDX), electrical resistance measurements and transport critical current density measurements. The zero-resistance temperature, $T_{c \text {-zero }}$ was found to decrease from $95 \mathrm{~K}$ to $84 \mathrm{~K}$ with the increase of graphene. The temperature dependence transport critical current density $\left(J_{c}\right)$ of the pure and graphene added bulk samples were investigated. $J_{c}$ of the non-added bulk sample was $1320 \mathrm{~mA} / \mathrm{cm}^{2}$ at $30 \mathrm{~K}$ while the $J_{c}$ of sample with $0.001 \mathrm{wt} . \%$ graphene was $3660 \mathrm{~mA} / \mathrm{cm}^{2}$ at $30 \mathrm{~K}$. Results showed that the $J_{c}$ of the Tl-1212 samples decreased with increasing graphene addition. Graphene acted as impurity which is believed to perform the flux pinning effect to Tl-1212. Thus, the $J_{c}$ of Tl-1212 superconductors was enhanced. The phase formation and morphology of samples Tl-1212 were also discussed in this paper.
\end{abstract}

Keyword: Superconductivity; Tl-1212 Superconductor; Transport Critical Current Density; Graphene 